\title{
Tsunami evacuation facility choice behavior model in flat area and rias area considering possibility to remain at home
}

\author{
Hiroyuki Yoshihara, Tatsuya Kishimoto \\ Graduate School of Science and Technology, Keio Univ. \\ Yokohama City, Japan
}

\begin{abstract}
The tsunami associated with the Great East Japan Earthquake that occurred on March 11, 2011 caused enormous damage to the coast of the Pacific Ocean. Since the occurrence of the Great Earthquake, it is urgent to develop tsunami evacuation countermeasures against the great tsunamis caused by the large earthquake that is expected to occur in the future in the country and coastal municipalities. In this study, we used logit model to estimate each parameters about the relationship between evacuees and buildings in flat area and rias area by using the evacuation behavior survey data of the Great East Japan Earthquake in 2011. The estimated results show that "whether the evacuation destination is home or not" is important when people choose the tsunami evacuation destination. Moreover, by comparing the moving in flat area and in rias area the difference between them becomes clear. By using these models, it enables to estimate the home selection probability in flat area and rias area.
\end{abstract}

Keywords: Tsunami, Evacuation behavior, Tsunami evacuation facility, Logit Model

\section{Research background and purpose}

The Great Tsunami associated with the Great East Japan Earthquake that occurred on March 11, 2011 caused enormous damage to the coast of the Pacific Ocean. Since the occurrence of the Great East Japan Earthquake, it is urgent to develop tsunami evacuation countermeasures against the tsunami caused by the large earthquake that is expected to occur in the future in the country and coastal municipalities.

A tsunami evacuation plan was formulated on the premise of evacuation to the nearest facility on the land side by foot. [1] However, it is clear that some people evacuate by car or stay at home without choosing to evacuate from the survey targeting Ojimachi City, Chiba Prefecture and Natori City, Miyagi Prefecture. [2] [3] In addition, there were characteristics of the evacuation destination selection due to differences in topography such as flat and rias. [4] The purpose of this research is to clarify the characteristics of the actual evacuation behavior of the coastal community residents at the time of the occurrence of the tsunami, by modeling the evacuation facility choice behavior of evacuees at the time of the Great East Japan Earthquake.

Specifically, we modeled the actual evacuation facility selection behavior at the time of the Great East Japan Earthquake using a multinomial logit model, and identified influential factors. Then, we calculated the home selection probability and clarified the evacuation difficulty area. We analyzed these in the topography of the flat area and the rias area and applied it to the regions where there is a possibility of the tsunami damage in the future, which helps the tsunami evacuation plan. Therefore, it is significant to compare and analyze the results.

\section{Evacuation behavior in target area}

The target areas are areas having sufficient number of samples for each municipality. In flat areas, they are Sendai City, Natori City, Iwanuma City, Watari-cho, Yamamoto-cho, and in rias area they are Ofunato City, Rikuzentakada City, Kesennuma City. The evacuation behavior data for modeling the evacuation behavior to the evacuation facility are GIS data of "Reconstruction Support Survey Archive". 
These are the point data of the evacuation place obtained by the questionnaire survey by the investigator's interview, and the polyline data of the evacuation route on the day of the Great East Japan earthquake disaster. The interview is carried out by the Ministry of Land, Infrastructure and Transport. The data set includes 20,503 routes of 10,603 people who suffered flood damage by the tsunami in 49 municipalities in 6 prefectures of Aomori, Iwate, Miyagi, Fukushima, Ibaraki and Chiba. Fig. 1 shows target areas in flat area and in rias area.

In order to create models with high versatility, we defined subjects for analysis as follows. We focused on evacuation behaviors in the coastal area by restricting the start of movement to within $1 \mathrm{~km}$ from the tsunami inundation area. We focused on the pure tsunami evacuation behavior by limiting the purpose of movement to "for evacuation from the tsunami" and "to go to shelter ". We focused on evacuees who move by walking and by car, since $90 \%$ or more of people walked or used a car on the day of the Great East Japan earthquake disaster. We eliminated the secondary evacuation after the tsunami by limiting the end time of the evacuation to the time before the tsunami maximum wave arrival time. The end of the movement was limited to home, designated evacuation shelters, facilities where more than two evacuated, since samples evacuated to a place that only a specific individual recognizes as an evacuation destination, such as an office or an acquaintance house among the evacuees of the tsunami, is not highly versatile as compared with other destinations Therefore, in flat area, 439 routes are defined as above (route 100 of Sendai city, 128 routes of Natori City, 51 routes of Iwanuma City, 80 routes of Watari-cho, 80 routes of Yamamoto-cho) and, in rias area, 359 routes are defined as above ( 89 routes of Ofunato City, 49 routes of Rikuzentakada City, 221 Route of Kesennuma City).

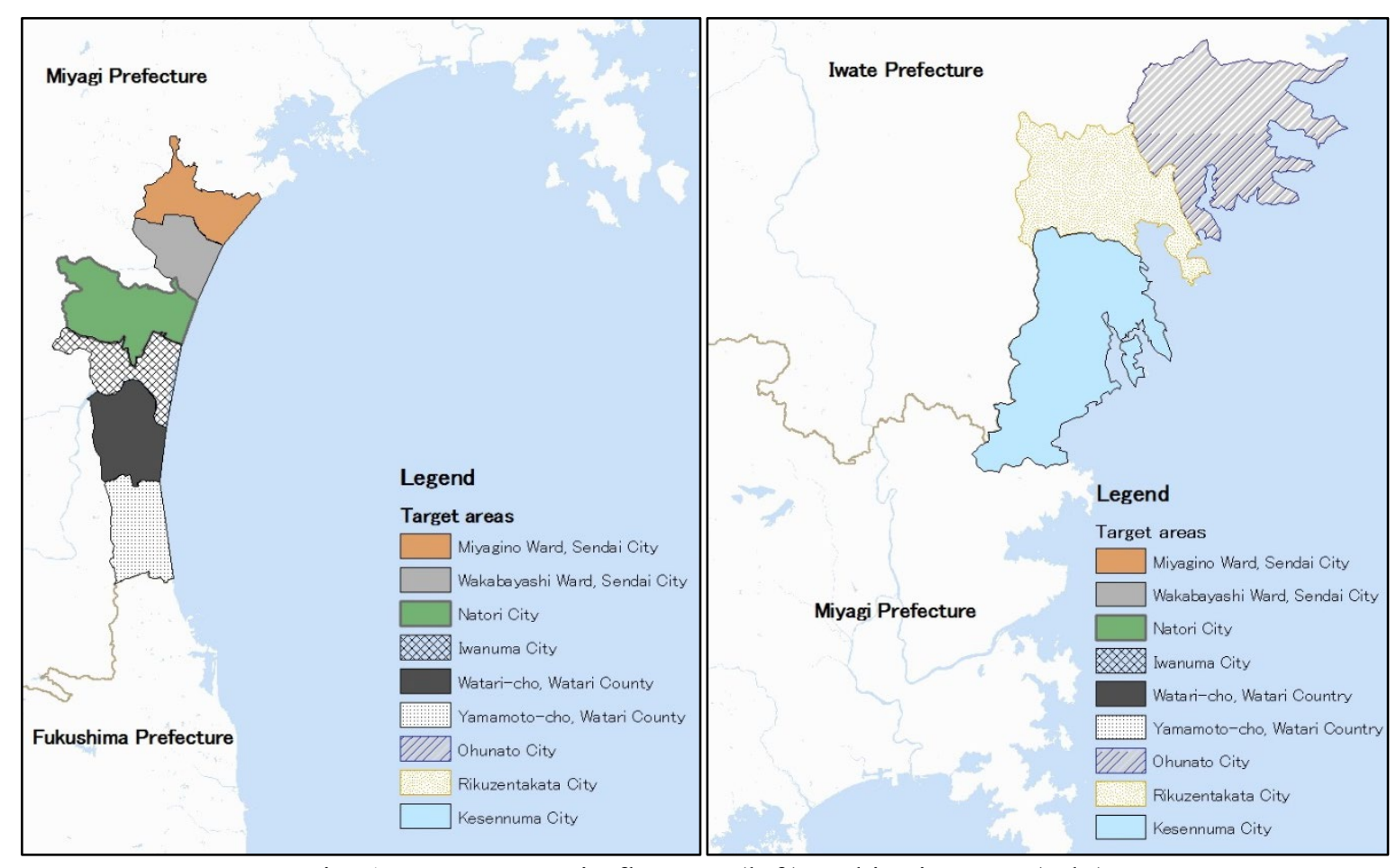

Fig. 1: Target areas in flat area (left) and in rias area (rght)

\section{Evacuation behavior in target area}

We estimate parameters of utility function using a multinomial logit model. The selection probability $P_{i j}$ and the utility $U_{i j}$ of the utility function are defined as follows when each evacuee selects the evacuation facility as the evacuation destination. 
We consider the behavior that individual $i$ select target $j$ of $J$ selection targets. The utility $U_{i j}$ is expressed as Equation (1) when each object $j$ is selected by individual $i$. At this time, each individual $i$ selects the largest utility among the objects. However, the utility $U_{i j}$ is not a definite value, but it varies in various situations.

$$
U_{i j}=V_{i j}+e_{i j}
$$

$V_{i j}$ is the utility function, and $e_{i j}$ is the probabilistic variable. The logit model assumes that the probability term $e_{i j}$ follows the Gumbel distribution, and the probability $P_{i j}$ that individual $i$ selects object $\mathrm{j}$ can be expressed by Equation (2).

$$
P_{i j}=\frac{\exp \left(V_{i j}\right)}{\sum_{j=1}^{J} \exp \left(V_{i j}\right)}
$$

The utility function $V_{i j}$ is determined by 1) the evacuation direction for the coastline, 2) the evacuation direction for the riverbank line, 3 ) the evacuation distance from the refugee to the evacuation facility, 4) the construction area of the evacuation facility, 6) the altitude of the evacuation destination, 7) the designated evacuation shelter dummy, and 8) the home dummy. Table. 1 shows factors to consider in determining $V_{i j}$.

In this research, we consider the psychological utility $\alpha_{1} \sin \theta_{i w j}, \alpha_{2} \sin \theta_{i c j}$ due to the relationship between the direction of the tsunami's attack and the evacuation direction. Assuming that the tsunami attacks from the sea side, we consider the psychological utility so that the utility is high in evacuation to the land side in the opposite direction and the utility is low in the case of evacuation to the sea side. Specifically, we set the angle between the coastline and the straight line from evacuee $i$ to evacuation facility $j$ as $\theta_{i w j}$ in case of evacuation on foot and $\theta_{i c j}$ in case of evacuation by car. $\alpha_{1}$ and $\alpha_{2}$ are coefficients.

As the tsunami runs along the river, we consider the psychological utility $\beta_{1} \sin \varphi_{i w j}, \quad \beta_{2} \sin \varphi_{i c j}$ due to the evacuation direction from the river. In the same way as the evacuation direction from the ocean, specifically, the angle between the river line and the straight line from the evacuee $i$ to the evacuation facility $j$ is set to $\varphi_{i w j}$ in case of evacuation on foot and $\varphi_{i c j}$ in case of evacuation by car. $\beta_{1}$ and $\beta_{2}$ are coefficients. Here, $\theta_{i w j}, \theta_{i c j}, \varphi_{i w j}$, and $\varphi_{i c j}$ are the angle $\left(-90^{\circ} \leqq \theta \leqq 90^{\circ}\right)$ formed by the straight line from the evacuee to the evacuation facility and the parallel line from the evacuee to the coastline or the river line, so the utility value becomes the maximum at 90 degrees and becomes the minimum at -90 degrees.

Since it is desirable to complete the evacuation as soon as possible from the occurrence of the earthquake, we consider the psychological utilities $\gamma_{1} D_{i w j}, \gamma_{2} D_{i c j}$ due to the evacuation distance from the evacuees to the evacuation facility. The evacuation distance on walking is set as $D_{i w j}(\mathrm{~m})$, the evacuation distance by car is set as $D_{i c j}(\mathrm{~m}) \cdot \gamma_{1}$ and $\gamma_{2}$ are coefficients. Here, the evacuation distance is defined as the straight distance between the evacuation start point and the evacuation arrival point.

Since the planar size of the evacuation facility is thought to affect the evacuation facility choice behavior, we set the utility $\delta \ln \left(S_{j}\right)$ by the building area $S_{j} . \delta$ is a coefficient. Since the vertical height of the evacuation facility is thought to affect the evacuation facility choice behavior, we set the utility $\operatorname{cln}\left(F_{j}\right)$ by the building floor $F_{j} . \varepsilon$ is a coefficient. In this case, taking into consideration the characteristic that the utility value is nonlinear, we use natural logarithm of the building area and the building floor in the model expression. [5]

When evacuating from the tsunami, it is important to evacuate to a place with high altitude. Therefore, we set the utility $\mu A_{j}$ by the altitude $A_{j}$ of the evacuation destination. $\mu$ is a coefficient.

Whether it is a designated evacuation facility set by each municipality is an important factor. Therefore, we introdyce the dummy variable $R_{j}$. It is 1 if the evacuation destination is a designated evacuation facility, otherwise it is 0 , and we set the utility $\rho R_{j} . \rho$ is a coefficient. 
In evacuation behaviors during the Great East Japan Earthquake, whether a refugee is at home is an important factor in the evacuation, since a certain number of evacuees have chosen their homes as evacuation destinations. Therefore, we introduce a dummy variable $H_{j}$. It is 1 if the evacuation destination is at home, otherwise it is 0 , and we set the utility $\sigma H_{j} . \sigma$ is a coefficient. Since the attribute at home differs depending on the evacuees, when $H_{j}$ is 1 , we set all other utility values is 0 . the utility function $V_{i j}$ is determined whether the evacuation destination is at home or not.

By using the above factors variables, we formulated the utility function $V_{i j}$. It can be expressed by Equation (3).

$$
V_{i j}=\left\{\begin{array}{c}
\alpha_{1} \sin \theta_{i w j}+\beta_{1} \sin \varphi_{i w j}+\gamma_{1} D_{i w j} \\
+\delta \ln \left(S_{j}\right)+\varepsilon \ln \left(F_{j}\right)+\mu A_{j}+\rho R_{j} \text { (by walking) } \\
\alpha_{2} \sin \theta_{i c j}+\beta_{2} \sin \varphi_{i c j}+\gamma_{2} D_{i c j} \\
+\delta \ln \left(S_{j}\right)+\varepsilon \ln \left(F_{j}\right)+\mu A_{j}+\rho R_{j} \text { (by car) } \\
\sigma H_{j} \text { (home evacuation) }
\end{array}\right.
$$

We obtained estimated values (maximum likelihood estimate) of the coefficients $\alpha_{1}, \alpha_{2}, \beta_{1}, \beta_{2}, \gamma_{1}, \gamma_{2}$, $\delta, \varepsilon, \mu, \rho, \sigma$ from the data on the evacuation facility selection by GIS data of "Reconstruction Support Survey Archive". The estimated value is a coefficient that maximizes the simultaneous occurrence probability $L^{*}$ by Equation (4). To maximize $L^{*}$ is equivalent to maximizing $L=\ln L^{*}$, so we obtained the value that maximizes $L$.

$$
L=\ln L^{*}=\sum_{j=1}^{J} \ln P_{i j}
$$

\begin{tabular}{|c|c|c|}
\hline Factors & Transportations & Utility function \\
\hline \multirow{2}{*}{ Direction from the $\operatorname{sea}[\sin \theta]$} & Walking & $\alpha_{1} \sin \theta_{i w j}$ \\
\hline & Car & $\alpha_{2} \sin \theta_{i c j}$ \\
\hline \multirow{2}{*}{ Direction from the river $[\sin \varphi]$} & Walking & $\beta_{1} \sin \varphi_{i w j}$ \\
\hline & Car & $\beta_{2} \sin \varphi_{i c j}$ \\
\hline \multirow{2}{*}{ Distance $[\mathrm{m}]$} & Waking & $\gamma_{1} D_{i w j}$ \\
\hline & Car & $\gamma_{2} D_{i c j}$ \\
\hline Facility area[LN $\left.\left(\mathrm{m}^{2}\right)\right]$ & . & $\delta \ln \left(S_{j}\right)$ \\
\hline Facility floor[LN(floor)] & 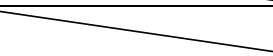 & $\varepsilon \ln \left(F_{j}\right)$ \\
\hline Facility altitude $[\mathrm{m}]$ & - & $\mu A_{j}$ \\
\hline Designated evacuation facility dummy $(0,1)$ & & $\rho R_{j}$ \\
\hline Home dummy $(0,1)$ & 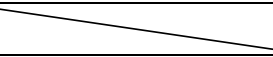 & $\sigma H_{j}$ \\
\hline
\end{tabular}

Table. 1 Factors to consider in modeling

In addition to the estimated value of the coefficient, we tested the estimation model by the $t$ value, the likelihood ratio $\rho^{2}$, and the hit rate as well. [6]

Table. 2 shows the estimation results of the parameters. In order to raise the hit rate, we eliminated parameters with low significance level by setting $\beta_{1}, \beta_{2}$ in the flat area model to 0 and $\alpha_{1}, \beta_{2}$ in the rias area model to 0 . In the flat area, the likelihood ratio is 0.547 , the hit rate is $53.2 \%$, in the rias area the likelihood ratio is 0.521 , the hit rate is $13.5 \%$. In addition, all the estimated parameters satisfy the 
significance level of $10 \%$. Both the flat area and the rias area model are accurate models in terms of the likelihood ratio, the hit rate and the significance level of each parameter.

$\alpha_{1}, \alpha_{2}, \beta_{1}, \delta, \varepsilon, \varepsilon, \mu, \rho, \sigma$ are positive values and $\gamma$ is negative for both flat and rias area. We found that people tended to choose the designated facility which is close and with high altitude and floor, large area, and away from the sea and the river or home according to situations. In the comparison between the flat area and the rias area, ,we can understand that the parameter of the evacuation direction in the flat area is larger than that in the rias area from the values of $\alpha_{1}, \alpha_{2}$, and $\beta_{1}$, and it influences the evacuation facility choice behavior. Moreover, we can understand that the importance of the designated evacuation facility and the home is greater in the flat area than in the rias area from the values of $\rho$ and $\sigma$, and it is easier to select the designated evacuation facility and the home as the evacuation destination.

Table. 2 Modeling results

\begin{tabular}{|c|c|c|c|c|c|c|}
\hline Transportations & Factors & Parameters & \multicolumn{2}{|c|}{ Flat areas } & \multicolumn{2}{|c|}{ Rias areas } \\
\hline Walking & \multirow{2}{*}{ direction from the $\operatorname{sea}[\sin \theta]$} & $\alpha_{1}$ & 1.45 & $* * *$ & & \\
\hline Car & & $\alpha_{2}$ & 1.35 & $* * *$ & 0.305 & ** \\
\hline Walking & \multirow{2}{*}{ direction from the river $[\sin \varphi]$} & $\beta_{1}$ & & 0.127 & $* *$ \\
\hline Car & & $\beta_{2}$ & & & & \\
\hline Walking & \multirow{2}{*}{ distance $[\mathrm{m}]$} & $\gamma_{1}$ & -0.00349 & $* * *$ & -0.00354 & $* * *$ \\
\hline Car & & $\gamma_{2}$ & -0.000990 & $* * *$ & -0.00238 & $* * *$ \\
\hline & facility area $\left[\mathrm{LN}\left(\mathrm{m}^{2}\right)\right]$ & $\delta$ & 0.105 & $*$ & 0.224 & $* * *$ \\
\hline & facility floor[LN(floor)] & $\varepsilon$ & 0.303 & $*$ & 0.248 & * \\
\hline & facility altitude[m] & $\bar{\mu}$ & 0.0385 & $* * *$ & 0.0268 & $* * *$ \\
\hline & designated evacuation facility dummy $(0,1)$ & $\rho$ & 1.25 & $* * *$ & 0.734 & $* * *$ \\
\hline & home dummy $(0,1)$ & $\sigma$ & 4.25 & $*$ & 2.30 & * \\
\hline \multicolumn{3}{|c|}{ Likelihood ratio $\left(\rho^{2}\right)$} & \multicolumn{2}{|l|}{0.547} & \multicolumn{2}{|c|}{0.521} \\
\hline \multicolumn{3}{|c|}{ Hitting percentage $(\%)$} & \multicolumn{2}{|l|}{53.2} & \multicolumn{2}{|l|}{13.5} \\
\hline
\end{tabular}

\section{Estimation of home selection probability}

Using the model, we estimated the home selection probability of each mesh divided into target areas by $100 \mathrm{~m}$. Despite the fact that home evacuation is dangerous in the evacuation behavior of the tsunami, since the utility of home selection is large in the actual evacuation behavior, it is very important to estimate the home selection probability. Fig. 2 shows the home selection probability and death rate of the flat area. The correlation coefficient of Pearson between the home selection probability and the death rate in the target area in the flat area is 0.428 , and it is a considerably positive correlation. If the mortality rate can be calculated in smaller unit than the postal code area, the correlation coefficient would become larger. According to Fig. 2, the home selection probability exceeds $40 \%$ in the area facing the ocean, and the death rate also has a high value of $10 \%$ or more there. It is considered as one reason that many designated evacuation facilities are located in places far from the sea. We think that it is necessary to install a tsunami temporary evacuation building etc. that can withstand high tsunamis in the coastal area.

Fig. 3 shows the home selection probability and death rate of the rias area. The correlation coefficient of Pearson between the home selection probability and the death rate in the target area in the rias area is 0.291 , and it is a slight positive correlation. Therefore, even in the rias area, areas with high home selection probability tend to be easily damaged by the tsunami. As you can see from Fig. 3, unlike the flat area, the home selection probability is higher near the river at upstream areas of the river than the area facing the sea. This means that the arrangement of designated evacuation facilities is not catching up 
with the tsunami going up highly along the river in the rias area. Therefore, it is necessary to install a tsunami temporary evacuation building along not only the coastal area but also the river.

In Chapter 3, the actual tsunami evacuation behavior at the time of the Great East Japan Earthquake can be modeled by dividing it into two topographies of the flat area and rias area. We apply this model to areas where tsunami damage is expected due to the Nankai Trough Earthquake. The Nankai Trough Earthquake is a large earthquake that is expected to occur in Japan in the future. The target areas are Kamakura City, Kanagawa Prefecture facing the Sagami Bay in the flat area and Owase City, Mie prefecture facing Owase Bay in the rias area. The population and the predicted tsunami height due to the Nankai Trough Earthquake in Kamakura City and Owase City is similar to those in the target areas in the Great East Japan Earthquake. From Fig. 4, in Kamakura City, home selection probability is high on both sides of Sagami Bay coast. From Fig. 4, in Owase City, the home selection probability is high at the inundation area along the Owase Bay coast and along the river. Tsunami evacuation facilities need to be installed to the upstream part of the river, considering the characteristic that not only the coast but also the tsunami goes up along the river. Since the distribution of home selection probability is similar to the features of the target areas in flat area and the rias area at the time of the Great East Japan Earthquake, we think that measures need to be taken promptly.
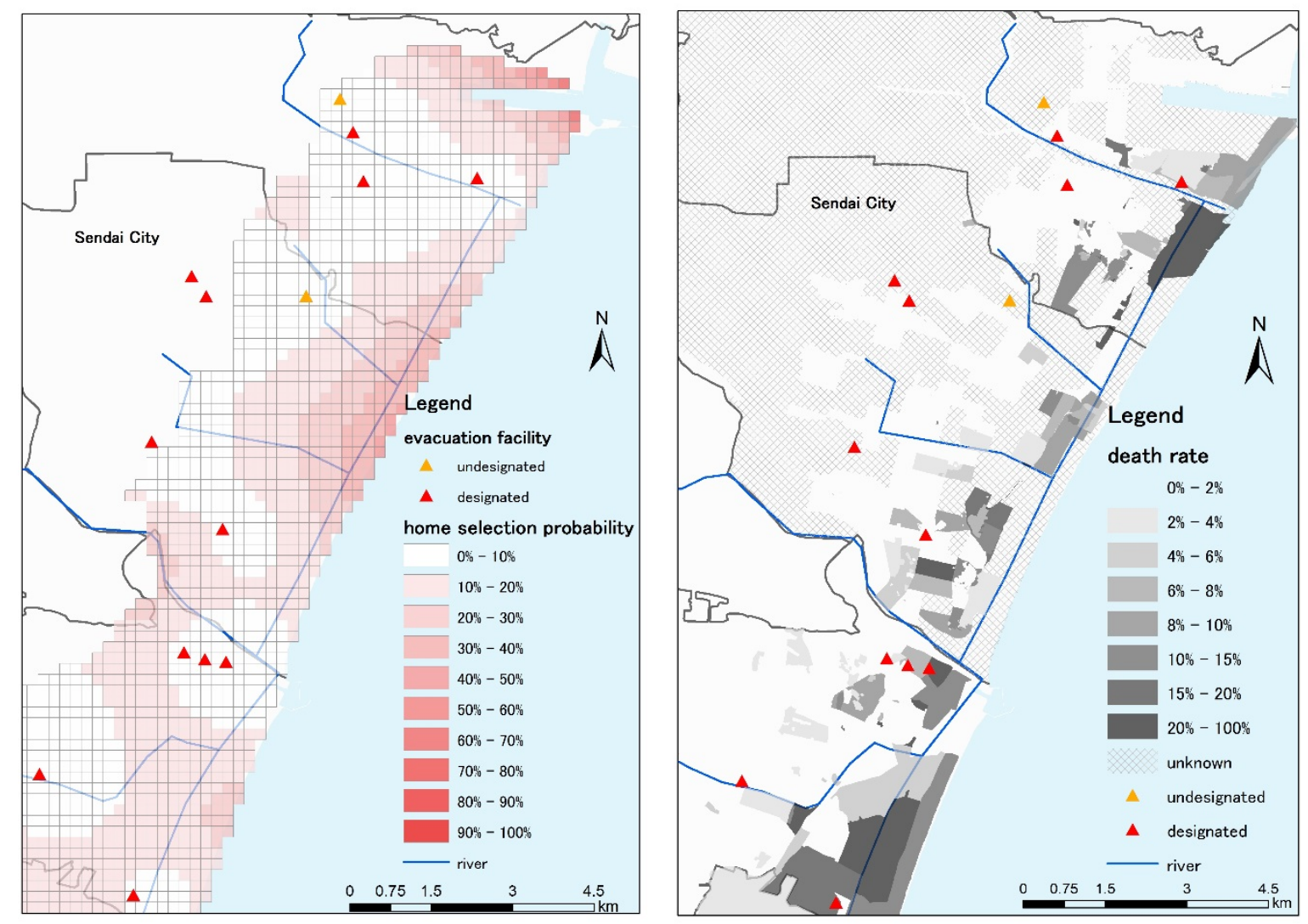

Figure. 2 Home choice probability (left) and death rate (right) in flat areas 

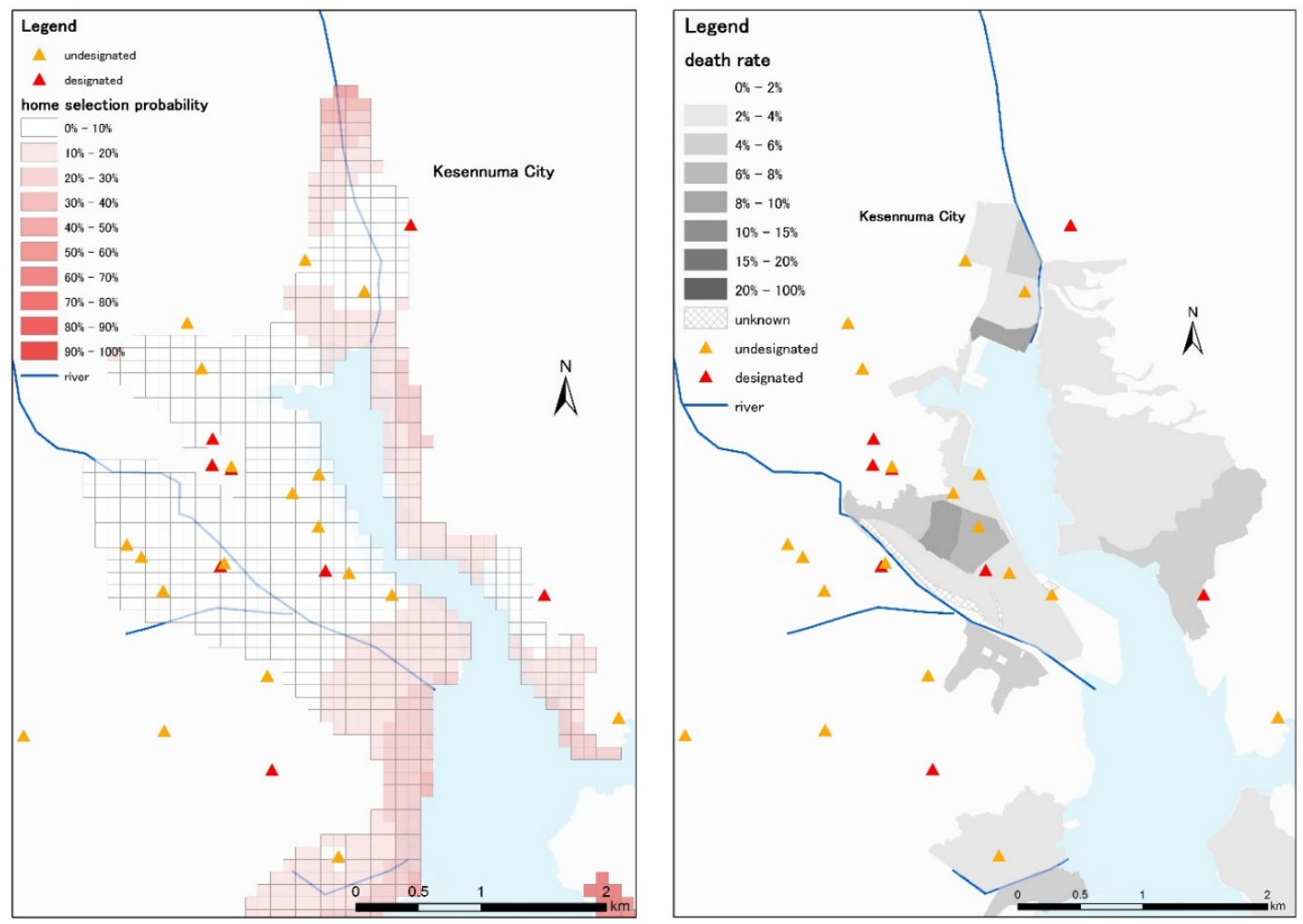

Figure. 3 Home choice probability (left) and death rate (right) in rias areas
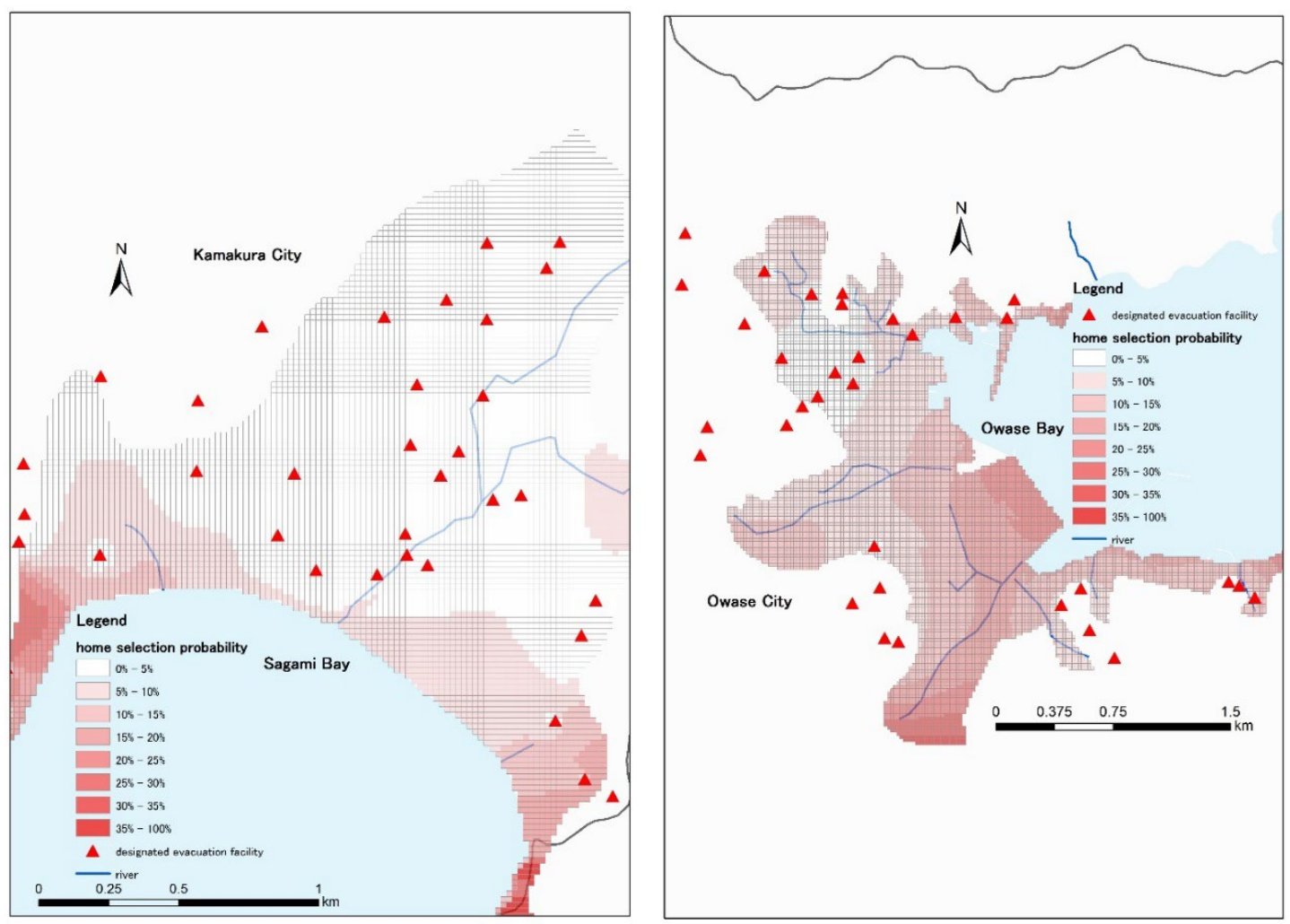

Figure. 4 Home choice probability in Kamakura (left) and in Owase (right) 


\section{Conclusion}

We analyzed the characteristics of the tsunami evacuation behavior by East Japan great earthquake by geography through a logit model with the reconstruction support survey archive.

In addition, we clarified evacuation difficulty areas through estimation of home selection probability. As a result, we were able to grasp where to evacuate facilities. We would like to make use of the findings obtained in this research to actual evacuation plans in preparation for the tsunami damage caused by the Nankai Trough massive earthquake.

As a future work, it is desirable to apply the findings obtained in this research to a wider range. Also, in this study, we proposed a region to expand evacuation facilities considering the home selection probability, but we have to simulate the case where the evacuation facility is newly established in the area and plan the placement method more quantitatively. In order to show how to arrange evacuation facilities in the evacuation plan, it is necessary to consider evacuation plans of evacuation behaviors and evacuation places / evacuation facilities. Furthermore, in planning the arrangement of evacuation facilities, it should be considered in relation to evacuation behaviors based on the characteristics of coast topography.

\section{References}

[1] T. Yamada, M. Sasaki, T. Kishimoto, "The sphere of evacuation facility based on the facility choice behavior model", AIJ J. Technol., vol. 22, no. 51, pp. 825-830, 2016

[2] T. Isagawa, "Residents' Attitude towards Tsunami Evacuation after the Great East Japan Earthquake Questionnaire surveys before and after the earthquake at Onjuku, Chiba prefecture which experienced small tsunami”, Institude Social Safety Science, vol. 12, no. 4, pp. 263-277, 2012

[3] H. Muurakami, "A study on fatality and factors affecting evacuation delay in Natori City hit by the 2011 Great East Japan Earthquake and Tsunami”, Journal of social safety science , vol. 24, pp. 101-110

[4] T. Yamada, T. Kishimoto, "Choice behavior model of refuge building selection during a tsunami coastal areas”, J. Archit. Plann., AIJ, vol. 80, no. 707, pp. 125-133, 2015

[5] R. Kimura, H. Hayashi, S. Tatsuki, K. Tamura, "Psychologically difined life reconstruction processes of disaster victims in the 1995 Hanshin-Awaji Earthquake", Journal of social safety science, vol. 6, pp. 241-250, 2004

[6] R. Alvarez, M. Camacho, G. Perez-Quiros, “Aggregate versus disaggregate information in dynamic factor models", International Journal of Forecasting, vol. 32, no. 3, pp. 680-694, 2016 